\title{
Filled and empty states of carbon nanotubes in water: Dependence on nanotube diameter, wall thickness and dispersion interactions
}

\author{
MALAY RANA and AMALENDU CHANDRA* \\ Department of Chemistry, Indian Institute of Technology, Kanpur 208016 \\ e-mail: amalen@iitk.ac.in
}

MS received 9 July 2007; accepted 1 August 2007

\begin{abstract}
We have carried out a series of molecular dynamics simulations of water containing a narrow carbon nanotube as a solute to investigate the filling and emptying of the nanotube and also the modifications of the density and hydrogen bond distributions of water inside and also in the vicinity of the outer surfaces of the nanotube. Our primary goal is to look at the effects of varying nanotube diameter, wall thickness and also solute-solvent interactions on the solvent structure in the confined region also near the outer surfaces of the solute. The thickness of the walls is varied by considering single and multi-walled nanotubes and the interaction potential is varied by tuning the attractive strength of the 12-6 pair interaction potential between a carbon atom of the nanotubes and a water molecule. The calculations are done for many different values of the tuning parameter ranging from fully Lennard-Jones to pure repulsive pair interactions. It is found that both the solvation characteristics and hydrogen bond distributions can depend rather strongly on the strength of the attractive part of the solute-water interaction potential. The thickness of the nanotube wall, however, is found to have only minor effects on the density profiles, hydrogen bond network and the wetting characteristics. This indicates that the long range electrostatic interactions between water molecules inside and on the outer side of the nanotube do not make any significant contribution to the overall solvation structure of these hydrophobic solutes. The solvation characteristics are primarily determined by the balance between the loss of energy due to hydrogen bond network disruption, cavity repulsion potential and offset of the same by attractive component of the solute-water interactions. Our studies with different system sizes show that the essential features of wetting and dewetting characteristics of narrow nanotubes for different diameter and interaction potentials are also present in relatively smaller systems consisting of about five hundred molecules.
\end{abstract}

Keywords. Hydrophobic hydration; hydrogen bonds; dispersion interactions; carbon nanotubes.

\section{Introduction}

The solvation characteristics of hydrophobic carbon nanotubes have been a subject of considerable interest in the recent past because of the relevance of such systems in chemistry, biology and materials science. The inner regions of water-filled nanotubes can be used to understand structural and dynamical behaviour of water confined in nanoscopic environments and can be used as simplified models for biological nanopores whereas the adsorption of water on the outer surfaces of the nanotubes can provide important information on hydrophobic interactions with nanoscopic surfaces. Therefore, it is not surprising that a number of experimental ${ }^{1-4}$ and theoretical ${ }^{5-18}$

We dedicate this work to Professor Debashis Mukherjee on his 60th Birthday. *For correspondence. studies have been devoted in the recent past to investigate these systems at a molecular level. In the theoretical studies of hydrophobic nanotubes, the cylindrical confining surface has either been modeled as a structureless smooth wall characterized by a purely repulsive $e^{5,6}$ or a combination of repulsive and attractive potentials ${ }^{6-8}$ or it has been modeled at a fully atomistic level with potential parameters usually chosen so as to mimic carbon nanotubes of different types. ${ }^{9-18}$ Although cylindrical pores with structureless surfaces are convenient model systems to investigate many of the generic aspects of solvation of these systems, the atomistic simulations have provided a more detailed picture of the hydration of hydrophobic nanopores, specifically carbon nanotubes in water. ${ }^{9-18}$ In some of these studies, the radii of the nanotubes were varied and different degrees of filling and solvation structures were found for the 
confined water. ${ }^{9-14}$ In these studies of varying pore radius, the carbon-water interactions were described by Lennard-Jones potential only and effects of varying interaction potentials were not explored. This is an important aspect of the solvation characteristics of these nanotubes because it has been shown very recently that water structure inside a $(6,6)$ narrow carbon nanotube can depend rather strongly on the strength of the attractive dispersion interactions between carbon and water molecules. ${ }^{15,16}$ Transitions from filled to empty states were when the well depth of carbon-water interactions was decreased by about a factor of half. However, these studies on the sensitivity of hydrophobic solvation on dispersion interactions were done only for a single nanotube of about $4 \cdot 1 \AA$ radius and thus it would be interesting to see if this sensitivity would also hold for nanotubes of different radii and lengths and also for different thermodynamic conditions.

In the present study, we make a detailed and systematic study of the solvation characteristics of carbon nanotubes of many different diameters, system sizes and carbon-water interaction potentials. Our primary focus is to find the existence of filled, partially filled and empty states of the nanotubes on variations of their radii and interaction potentials and how the hydrogen bonded structure of water outside the nanotubes differ from those inside the tubes. In addition, we have also looked at the effects of wall thickness on the solvation properties of these nanotubes. We note that the water molecules inside and outside a single-walled carbon nanotube is separated by a layer of carbon atoms of about $3 \AA$ thickness. Although such a single-layer of carbon atoms is sufficient for the disruption of hydrogen bond network and for the creation of cavity repulsion potential, water molecules are also known to have long range electrostatic interactions which mean interactions between water molecules inside and outside a single-walled nanotube may also play a role in determining the wetting and dewetting behaviour of these hydrophobic solutes. Such issues can be answered by increasing the wall thickness of the nanotubes. Compared to the case of a single-walled tube, water molecules on the outer side of a multi-walled tube would be further away from those inside the tube and so interactions between them would also be much weaker. Keeping this in mind, we have simulated the solvation of carbon nanotubes with different thicknesses, starting from single-walled to multiwalled and investigated how the solvation character- istics change with changes of thickness of the nanotube walls.

The present calculations reveal that the structure of water inside and near outer surface a carbon nanotube depends rather weakly on its wall thickness but depends rather strongly on its diameter and strength of the carbon-water dispersion interactions. Calculations with single and triple-walled nanotubes produce very similar density profiles and hydrogen bonded structure of water molecules inside and also outside the nanotubes. On the other hand, transitions from completely filled to completely empty states of the nanotubes are found on changing their diameters and also the attractive part of the solute-water interaction potential. This indicates that the solvation characteristics are primarily determined by the net effects of energy loss due to hydrogen bond network disruption, creation of cavity repulsion potential and offset of the same by attractive solute-water interactions. The long range electrostatic interactions between water molecules on the two opposite sides of the nanotube wall do not seem to make any significant influence on the overall solvation structure of these hydrophobic solutes. Our calculations with different system sizes show that the essential features of solvation properties, e.g. wetting and dewetting characteristics for different diameters and interaction potentials, are also present in relatively smaller systems consisting of about four hundred solvent molecules. Since essentially all of the existing simulations of water in carbon nanotubes are based on empirical pair potentials for carbon-water interactions and the true interaction potential is not known, the smaller systems of the present study can be employed for more accurate calculations involving $a b$ initio molecular dynamics methods to have a better understanding of the wetting and dewetting behaviour and overall solvation characteristics of carbon nanotubes in water.

We have organized the rest of the paper as follows. In §2, we describe the models of water and carbon nanotubes that are employed in the present work and also other details of simulations that we have carried out. In §3, we have presented the results of the density and hydrogen bond distributions of water in the confined regions inside the nanotubes and also in the vicinity of the outer surfaces of the nanotubes for different interaction potentials. Results are presented for different diameter and for both single and multi-walled nanotubes and also for different system sizes. Our conclusions are briefly summarized in $\S 4$. 


\section{Model and simulation details}

Our simulation systems consist of a narrow carbon nanotube immersed in liquid water. The diameter and the wall thickness of the nanotubes and also attractive strength of the nanotube-water interactions are varied over wide ranges. In all of our present simulations, the water molecules are represented by the well-known SPC/E model ${ }^{19}$ where the interaction between atomic sites of two water molecules is given by

$$
u_{w w}\left(r_{i}, r_{j}\right)=\left(\frac{A_{i j}}{r_{i j}}\right)^{12}-\left(\frac{B_{i j}}{r_{i j}}\right)^{6}+\frac{q_{i} q_{j}}{r_{i j}},
$$

where, $q_{i}$ is the charge of the $i$ th atom of a water molecule and $A_{i j}$ and $B_{i j}$ are the coefficients of the short range 12-6 interactions which are non-zero only for oxygen-oxygen interactions and zero for interactions with hydrogen atoms. For oxygen-oxygen interactions, the values of $A_{i j}$ and $B_{i j}$ are 3.428 $\left(\mathrm{kJ} \mathrm{mol}^{-1}\right)^{1 / 12} \AA$ and $3.7122\left(\mathrm{~kJ} \mathrm{~mol}^{-1}\right)^{1 / 6} \AA$, respectively. We note that these values of $A_{i j}$ and $B_{i j}$ correspond to a Lennard-Jones well-depth of $0.6502 \mathrm{~J} \mathrm{~mol}^{-1}$ and diameter of $3.169 \AA$. The partial charges on the oxygen and hydrogen atoms are $-0.8476 e$ and $+0.4238 e$, respectively, where $e$ is the magnitude of the electronic charge.

We have considered $(n, n)$ 'armchair'-type carbon nanotubes with $n$ is varied from 5 to 20 . This corresponds to a carbon-to-carbon diameter of $6.78 \AA$ for $n=5$ to $27 \cdot 12 \AA$ for $n=20$. The length of the nanotubes is $13.52 \AA$. For most of the systems, a singlewalled nanotube is considered which is immersed in a bath of more than 1000 water molecules. For some of the calculations, we have also considered larger systems of about 2000 water molecules containing a triple-walled nanotube with an inter-wall separation of $2.71 \AA$ and inner diameter of $8 \cdot 14 \AA$. Each layer is obtained by folding a graphite sheet with carbon atoms placed in hexagonal positions with a carboncarbon distance of $1.42 \AA$. The carbon atoms are kept fixed at their original positions so that only the carbon-water interactions and forces need to be considered in the present simulations. In the present work, we have considered uncharged hydrophobic nanotubes consisting of carbon atoms without any partial charges. For such a solute with uncharged carbon atoms, the most significant solute-water interactions will be the attractive dispersion interaction and the short-range repulsive interaction. Since the disper- sion interactions with hydrogen atoms are minimal, we consider only the oxygen atoms for carbonwater interaction and it is modeled by the following potential

$$
u_{c o}\left(r_{i}, r_{j}\right)=\left(\frac{A_{c o}}{r_{i j}}\right)^{12}-\lambda\left(\frac{B_{c o}}{r_{i j}}\right)^{6},
$$

where $A_{C O}=3.470 \quad\left(\mathrm{~kJ} \mathrm{~mol}^{-1}\right)^{1 / 12} \AA, \quad B_{C O}=3.666$ $\left(\mathrm{kJ} \mathrm{mol}^{-1}\right)^{1 / 6} \AA$, and $\lambda$ is a parameter that determines the weight of the attractive dispersion part of the carbon-oxygen interaction potential. We note that the potential of (2) can also be expressed in the following form

$$
u_{c o}\left(r_{i}, r_{j}\right)=4 \varepsilon_{c o}\left[\left(\frac{\sigma_{c o}}{r_{i j}}\right)^{12}-\lambda\left(\frac{\sigma_{c o}}{r_{i j}}\right)^{6}\right],
$$

with $\sigma_{C O}=3.2846 \AA$ and $\varepsilon_{\mathrm{CO}}=0.4837 \mathrm{~kJ} \mathrm{~mol}^{-1}$. If we set $\sigma_{C O}=\left(\sigma_{C}+\sigma_{O}\right) / 2$ and $\varepsilon_{C O}=\left(\varepsilon_{C} \varepsilon_{O}\right)^{1 / 2}$ and use SPC/E values for water Lennard-Jones parameters, i.e. $\sigma_{O}=3.169 \AA$ and $\varepsilon_{O}=0.6502 \mathrm{~kJ} \mathrm{~mol}^{-1}$, then for $\lambda=1$, the above potential becomes equivalent to a Lennard-Jones interaction between nanotubes carbon and water oxygen atoms with a well-depth parameter of $0.3598 \mathrm{~kJ} \mathrm{~mol}^{-1}$ and diameter of $3.4 \AA$ for carbon atoms. These Lennard-Jones parameter values correspond to AMBER force fields ${ }^{20}$ for a $s p^{2}$ carbon atom and have been used in many of the earlier studies of water near graphite surfaces and carbon nanotubes. In the present work, we have varied the values of $\lambda$ from 0.0 to 1.0 to tune the potential from a purely repulsive to a fully Lennard-Jones interaction and thus to study the role of the attractive dispersion component in the solvation of hydrophobic nanopores. We note that the potential of (3) with the tunable $\lambda$ is conformal with another Lennard-Jones potential with modified parameters: $\varepsilon_{C O}^{\prime}=\varepsilon_{C O} \lambda^{2}$ and $\sigma_{C O}^{\prime}=\sigma_{C O} / \lambda^{1 / 6} \cdot{ }^{16}$ Clearly, as the value of $\lambda$ is decreased, the well depth of interaction decreases and the effective diameter of the pair of atoms increases. This leads to a change in the slope of the initial repulsive part of the interaction with variation of $\lambda$ as can be seen from figure 1a where the carbon-oxygen interactions are plotted for three different values of $\lambda$. One way of making the carbon-oxygen interaction fully repulsive without altering the slope of its short-range part is to use the repulsive part of the Lennard-Jones potential according to the WeeksChandler-Andersen (WCA) decomposition scheme ${ }^{21}$ 


$$
\begin{aligned}
& u_{c o}{ }^{(W C A-R)}\left(r_{i j}\right)=4 \varepsilon_{c o}\left[\left(\frac{\sigma_{c o}}{r_{i j}}\right)^{12}-\lambda\left(\frac{\sigma_{c o}}{r_{i j}}\right)^{6}\right]+\varepsilon_{c o}, \\
& \text { for } r_{i j}<r_{m} ; \quad=0 \text { for } r_{i j} \geq r_{m}
\end{aligned}
$$

where $r_{m}=2^{1 / 6} \sigma$ is the distance at which the LennardJones potential has its minimum. We will call the above potential as WCA-repulsive or WCA-R.

First, we simulated the system of a $(6,6)$ nanotube of diameter $8 \cdot 14 \AA$ immersed in water for six different values of $\lambda$ ranging from 1.0 to 0 . The nanotube is placed at the centre of the simulation box with its cylindrical axis pointing along the $\mathrm{z}$ direction. Initially, 864 water molecules are equilibrated in a cubic box whose size is determined by the density of water at room temperature and then the nanotube is inserted into the water. Upon insertion of the nanotube, overlapping water molecules are removed from the simulation systems and the resultant system containing the nanotube is re-equilibrated for each value of the tuning parameter $\lambda$. During this period of re-equilibration and also during the subsequent production phase of the simulation run, the nanotube is kept fixed and only the water molecules are allowed to move. Subsequently, we carried out similar simulations for systems containing nanotubes of 8 different diameters, from $6 \cdot 78 \AA$ for $(5,5)$ to $27.12 \AA$ for $(20,20)$ type, immersed in bigger boxes of more than a thousand water molecules. In these simulations, each box contained 1372 molecules (2048 for the $(20,20)$ nanotube) to begin with
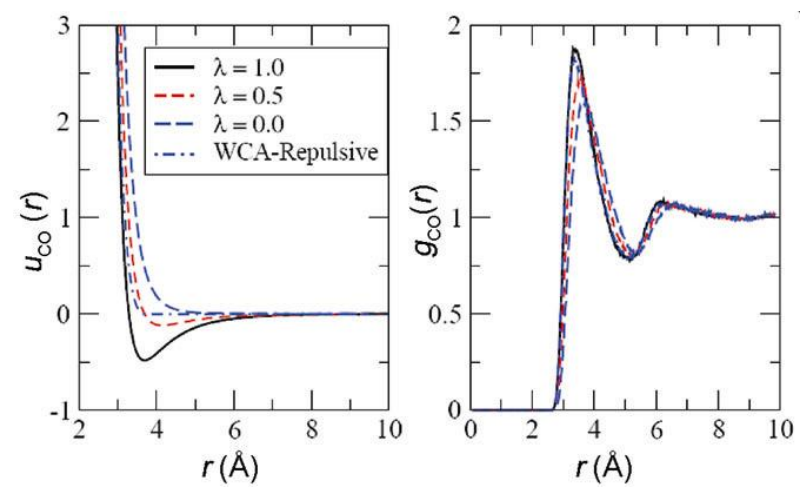

Figure 1. (a) The carbon-water interaction potential as given by (2) for three different values of the tuning parameter $\lambda$ and also for the WCA-repulsive potential. (b) The carbon-oxygen radial distribution functions obtained from simulations of a single carbon atom in water for different values of $\lambda$ and WCA-repulsive potential. The different curves in (b) are as in (a). and then the overlapping water molecules are removed and reequilibration was done. These simulations of nanotubes of varying diameters were done for $\lambda=1$ and 0 . In the next phase, we considered a triplewalled nanotube with an inner diameter of $8.14 \AA$ (the three walls are of $(6,6),(10,10)$ and $(14,14)$ type) characterized by $\lambda=1$ and $\lambda=0$ and also single-walled $(6,6)$ and $(12,12)$ nanotubes characterized by WCA-repulsive potentials. Simulations are also done for a single carbon atom immersed in 499 water for the same values of the $\lambda$ parameter and WCA-repulsive interactions. Finally, we simulated a smaller system containing a single-walled $(6,6)$ nanotube immersed in 455 water molecules (after removing the overlapping molecules) to see if the essential aspects of the salvation characteristics that are observed for larger systems are also exhibited by the smaller systems of few hundred molecules. These smaller system simulations are also done for two different values of $\lambda$ and thus, altogether, we have simulated thirty two different systems in the present study.

In the present simulations, we have employed a minimum image convention for the short-range interaction potential and the water-water long-range electrostatic interactions are treated using the Ewald method. ${ }^{22}$ We employed the quaternion formulation of the equations of rotational motion and, for the integration over time; we adapted the leap-frog algorithm with the time-step of $10^{-15} \mathrm{~s}(1 \mathrm{fs})$. For each system, we first ran the simulation for $100 \mathrm{ps}$ for equilibration during which the temperature was kept fixed at $298 \mathrm{~K}$ through rescaling of velocities. Upon equilibration, each system was further run for a production phase of 100-200 ps depending on system size with occasional re-scaling of velocities to maintain the average temperature of $298 \mathrm{~K}$ and we used this production phase of the simulation for calculation of the density profiles and hydrogen bond properties of water molecules in the confined region inside the nanotubes and also in the regions near the outer surfaces of the nanotubes.

\section{Results and discussions}

First, we discuss the effects of varying carbon-water dispersion interactions on the water structure around a carbon atom. In figure 1a, we have shown the carbon-water interaction potentials for three different values of the tuning parameter of the solute-water attractive potential: $\lambda=1 \cdot 0,0.5$ and 0 . In figure $1 \mathrm{~b}$, 
we have shown the corresponding carbon-oxygen radial distribution functions as obtained from simulations of a single carbon atom in water for these three different values of the tuning parameter. It is seen that in all cases, even when the carbon-water interaction is fully repulsive, there is enhanced density of water in the first hydration shell near the carbon atom as compared to that in the bulk phase. In particular, the density profiles are very similar for $\lambda=1.0$ and 0.5 . Even for the fully repulsive potentials for $\lambda=0$ and WCA-repulsive, the water structure around the small carbon atoms is not very different from those for $\lambda=1$. Thus, the attractive dispersion interaction plays only a minor role in determining the solvation structure of small hydrophobic solutes, their hydration structures are primarily determined by the repulsive part of the potential. On the contrary, when the solvation structures of carbon nanotubes made of many carbon atoms are concerned, dramatic differences are found for different values of the tuning parameter as can be seen from figure 2 . Here we have shown the density profiles of water in the region inside and also near the outer surfaces of
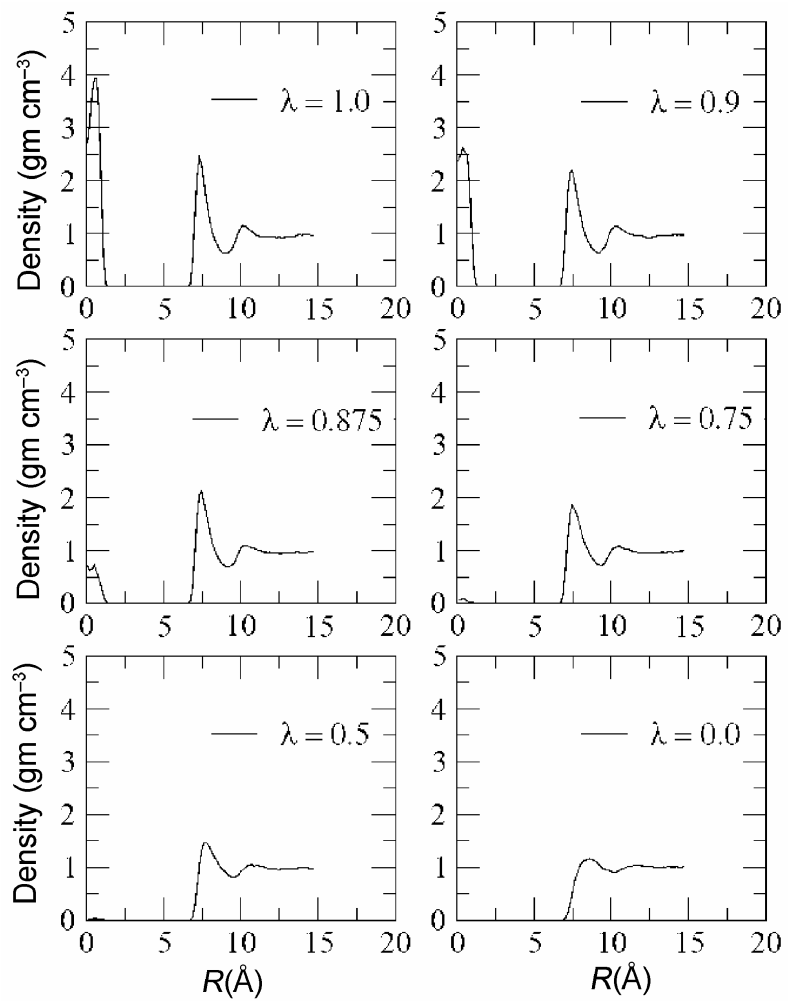

Figure 2. The density profiles of water inside and in the vicinity of the outer surface of a single-walled $(6,6)$ nanotube for different values of the tuning parameter $\lambda$. a $(6,6)$ nanotube with a diameter of $8.14 \AA$ and length $13.52 \AA$. The density profiles are computed from the simulation trajectory by taking a cylindrical slab around the centre of the simulation box with its axis and length the same as those of the nanotube and its radial dimension taken to be the half of the length of the simulation box along the z-direction. It is seen that, depending on the value of $\lambda$, very different density profiles are found both inside and also outside the surfaces of the nanotube. Especially, a complete dewetting is observed for the inner region for the fully repulsive potential and wetting is observed only when $\lambda=0.875$ or higher. Thus, when the strength of the atom-water attractive dispersion interaction is not too small, the collective effects of the large number of such dispersion interactions stabilize a few water molecules inside the nanotube and complete or partial filling of the nanotube is observed. The calculations with the WCA-repulsive potential produced very similar results which are discussed in the later part of this section. We note in this context that similar wetting and dewetting behaviour with changes of interaction potentials has also been observed for water between two hydrophobic graphite plates. ${ }^{23}$ In figure 3 , we have shown typical configurations taken from the simulation trajectories for two different values of $\lambda$. These snapshots clearly show the filled and empty states of the nanotube as well as different water densities near the outer surfaces of the nanotubes for varying attractive strength of the carbon-water interaction.

In figure 4, we have shown the effects of varying diameter of the nanotubes on their solvation characteristics for $\lambda=1$ and 0 . For the fully Lennard-Jones potential $(\lambda=1)$, the wetting of the tubes are seen for all the diameters except the smallest one of $(5$, $5)$. The $(5,5)$ nanotube has a diameter of $6 \cdot 78 \AA$ which does not appear to hold even a single water chain inside. Only a single strand of water is found inside the $(6,6)$ and $(8,8)$ nanotubes whereas, for the $(10,10)$ nanotube, a one-dimensional water strand along the nanotube axis and a two-dimensional layer of water molecules near the inner surface are found. For larger diameter nanotubes from $(12,12)$ to $(20$, 20 ), two or more layers of water molecules are found in the inside regions for the fully Lennard-Jones interaction potentials. Typical configurations corresponding to this one-dimensional and layered structure of water molecules inside nanotubes of varying diameters are shown in figure 5.

For the fully repulsive carbon-water interactions $(\lambda=0)$, the water structures are very different inside 

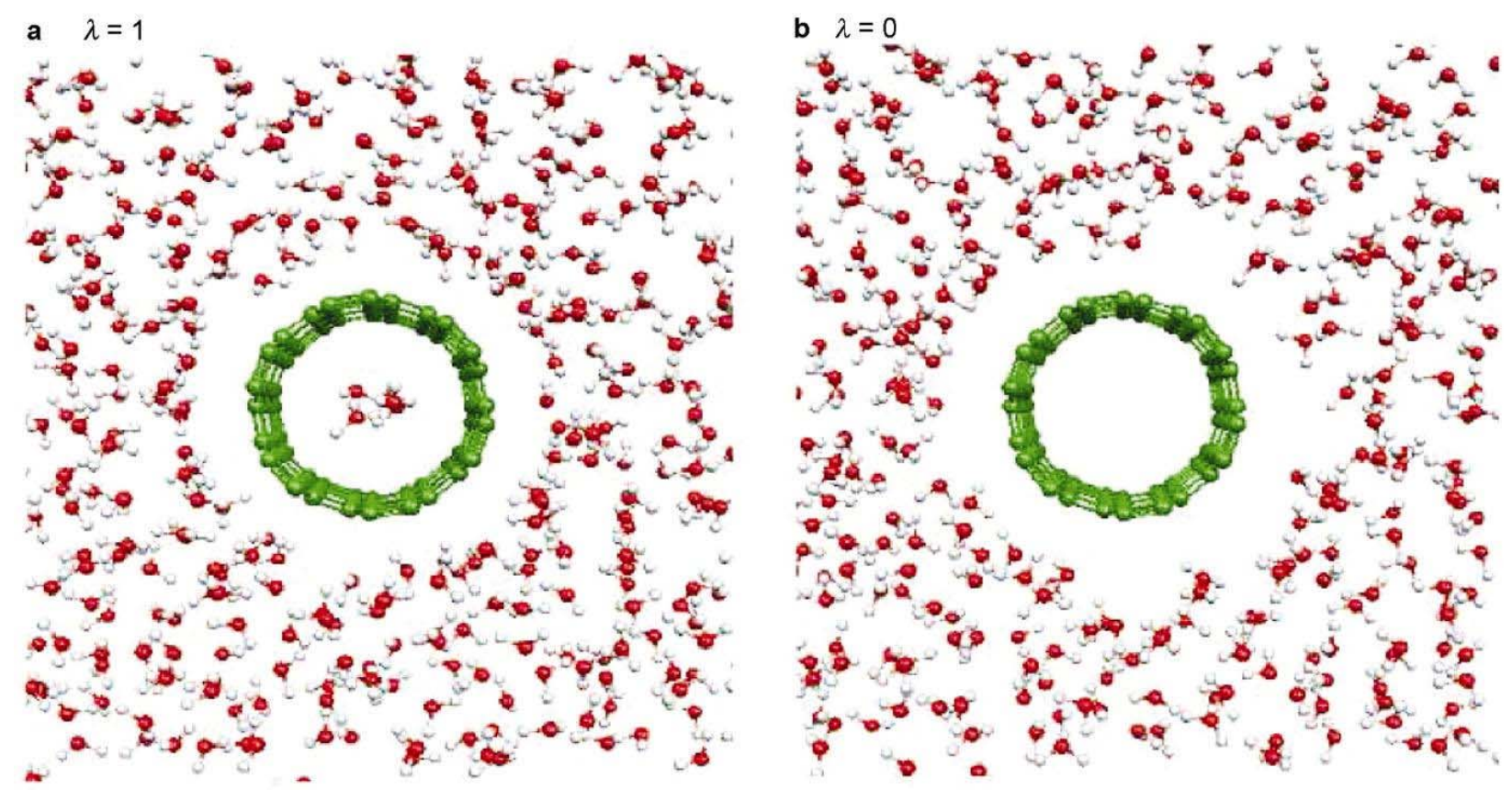

Figure 3. Snap-shots of configurations of water inside and in the vicinity of the outer surface of a single-walled $(6,6)$ nanotube for (a) $\lambda=1 \cdot 0$ and $\lambda=0$.
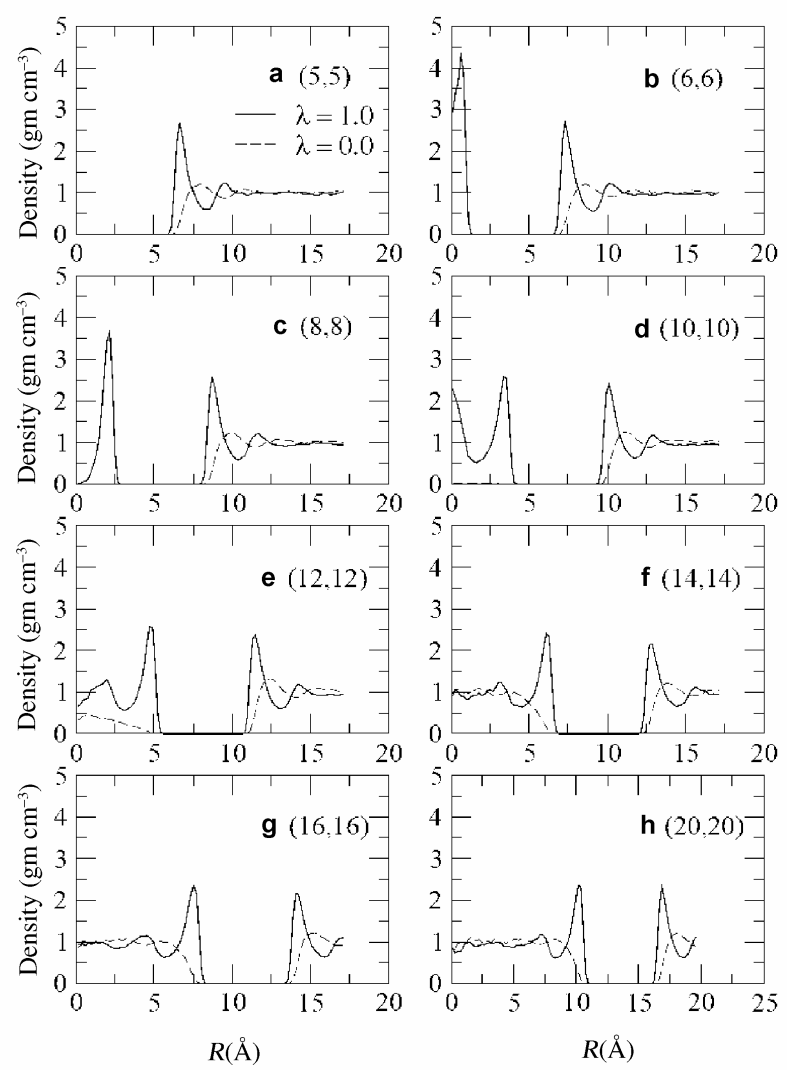

Figure 4. The density profiles of water inside and in the vicinity of the outer surfaces of single-walled $(n, n)$ nanotubes ( $n=5$ to 20) for $\lambda=1.0$ (solid curves) and $\lambda=0$ (dashed curves). the nanotubes and also near the outer surfaces. Here, complete dewetting is observed for the smallest diameter and also for the larger diameters of $(6,6),(8$, $8)$ and $(10,10)$ nanotubes. Only for $(12,12)$ and larger diameter nanotubes, filling of the confined region by water molecules is observed. Even then, the density profiles of water both inside and outside the nanotubes are found to be significantly different from those for $\lambda=1$. The hydrogen bond distributions of the systems of figure 4 are shown in figure 6 for both $\lambda=1.0$ and 0 . We have used a geometric definition of the hydrogen bonds as was done in earlier studies. ${ }^{23-25}$ We have, however, used only distance criteria and no angular cut-off was used. The number of hydrogen bonds per water molecules is found to be significantly reduced in confined region for the smaller diameter nanotubes having only a single one-dimensional water chain. Since the larger diameter nanotubes support both a water chain and also twodimensional water layers near the inner surfaces, the number of hydrogen bonds per water molecule also increases as compared to the case where only a single one-dimensional chain was present. Again, significant differences are found between the hydrogen bond distributions for fully Lennard-Jones and fully repulsive potentials, especially for the smaller diameter nanotubes.

In figure 7a, we have shown the effects of wall thickness on the wetting and dewetting behaviour of 
a narrow nanotube. In this figure, we have shown the water density profiles of water in the inside and outside regions of a single-walled and triple-walled nanotube with an inner diameter of $6.8 \AA$ for $\lambda=1$. The hydrogen bond distributions of water molecules inside the tubes and also near the outer surfaces are shown in figure $7 \mathrm{~b}$. The corresponding results of the density and hydrogen bond distributions for $\lambda=0$ are presented in figure 8 . Overall, the results for the single-walled nanotubes are found to be very close to those for the triple-walled for both values of the tuning parameter $\lambda$. Thus, the thickness of the nanotube walls seems to play a minor role in determining their overall hydration characteristics including the

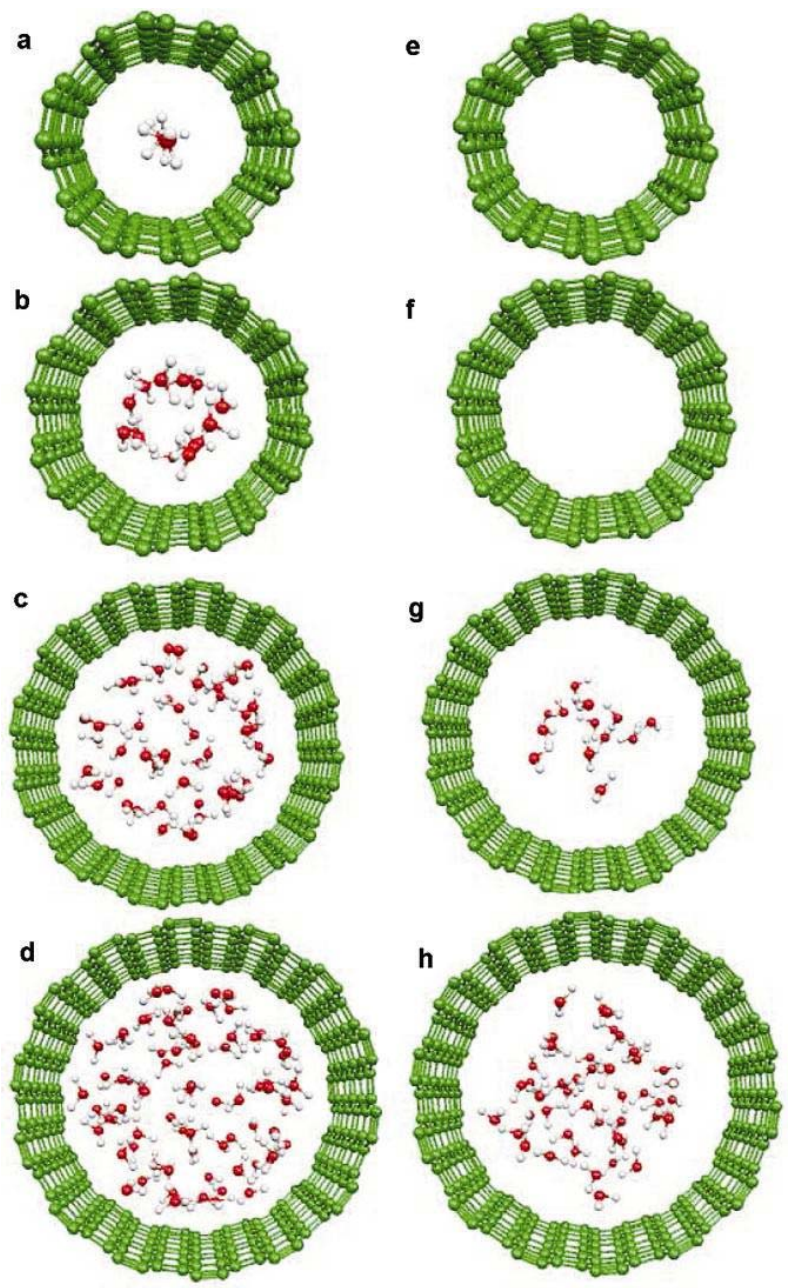

Figure 5. Snap-shots of configurations of water inside single-walled nanotubes of (a and e) $(6,6)$, (b and f) (8, 8), (c and g) $(12,12)$ and (d and h) $(14,14)$ types. The configurations in the left column are for $\lambda=1.0$ and those of the right column corresponds to $\lambda=0$. details of wetting versus dewetting behaviour and hydrogen bonded water structure near the nanotube walls.

In figure 9, we have compared the results for two fully repulsive potentials described by $\lambda=0$ (2) and WCA-repulsive potential (4). The WCA-repulsive potential was used earlier in studies of wetting and dewetting behaviour of nanoscopic graphite plates and thus it is important to see how the results for $\lambda=0$ compare with those of the WCA-repulsive potential. We have shown the results for two different nanotubes: $(6,6)$ and $(12,12)$. It is seen that both the potentials produce very similar results for water densities inside and outside the nanotubes. In particular, partial filling of the $(12,12)$ nanotube and complete emptying of the $(6,6)$ tube are reproduced by the both the potentials. Thus, the slight changes in the slopes of the short-range repulsive potential are not found to have any significant effects on the overall solvation features of these nanotubes, rather such features, especially with respect to their filled
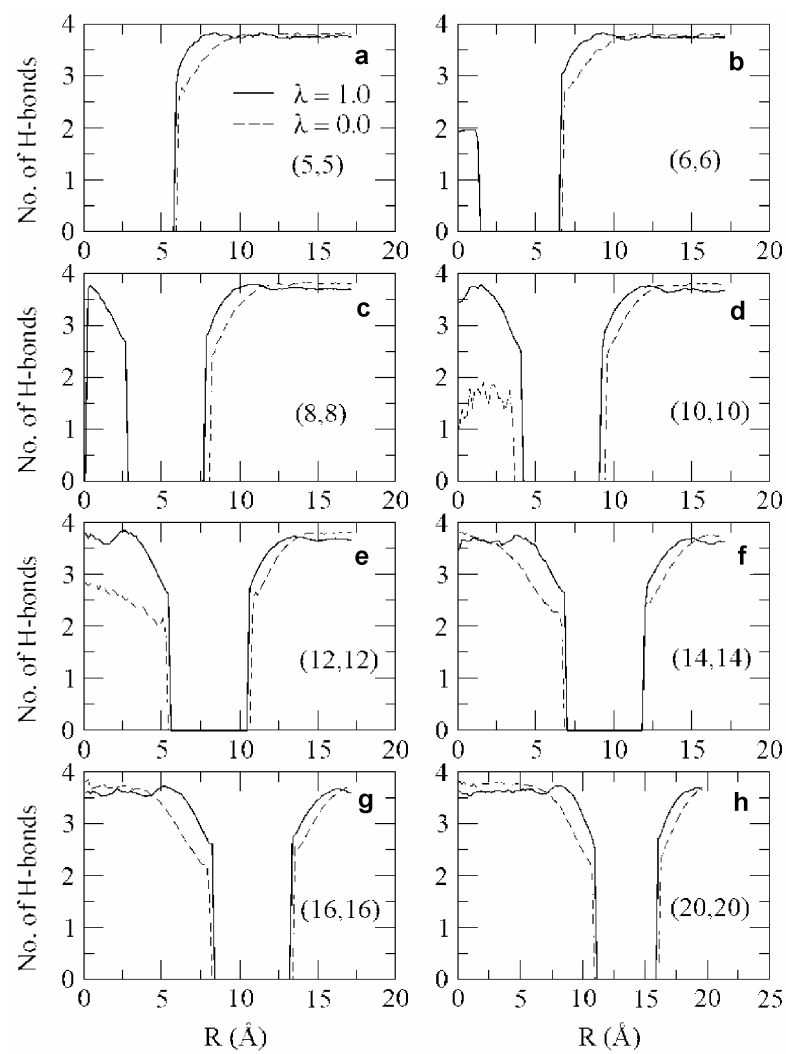

Figure 6. The number of hydrogen bonds per water molecule inside and in the vicinity of the outer surfaces of single-walled $(n, n)$ nanotubes $(n=5$ to 20$)$ for $\lambda=1 \cdot 0$ (solid curves) and $\lambda=0$ (dashed curves). 

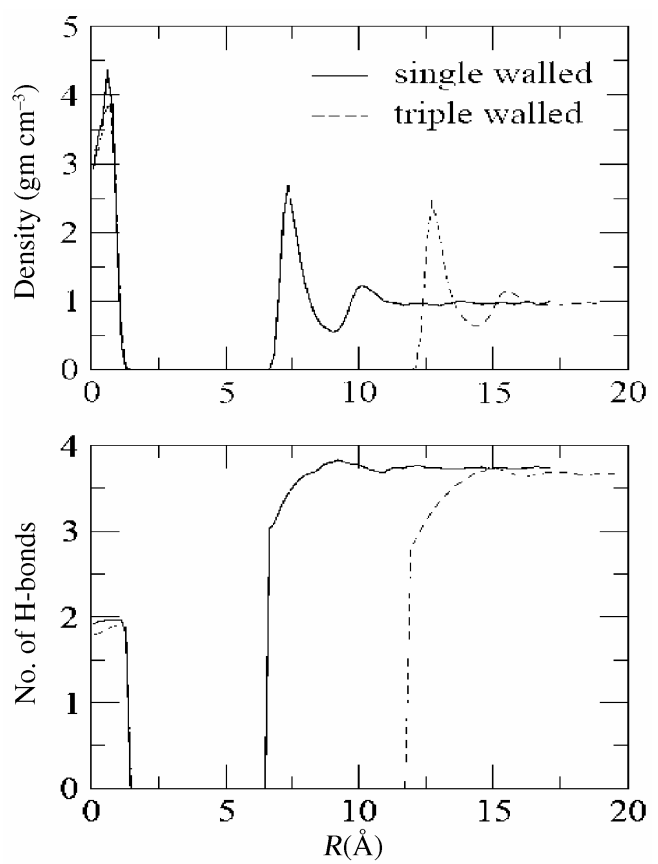

Figure 7. The density profiles and hydrogen bond distributions of water inside and in the vicinity of the outer surface of a single-walled and triple-walled $(6,6)$ nanotube for $\lambda=1$.
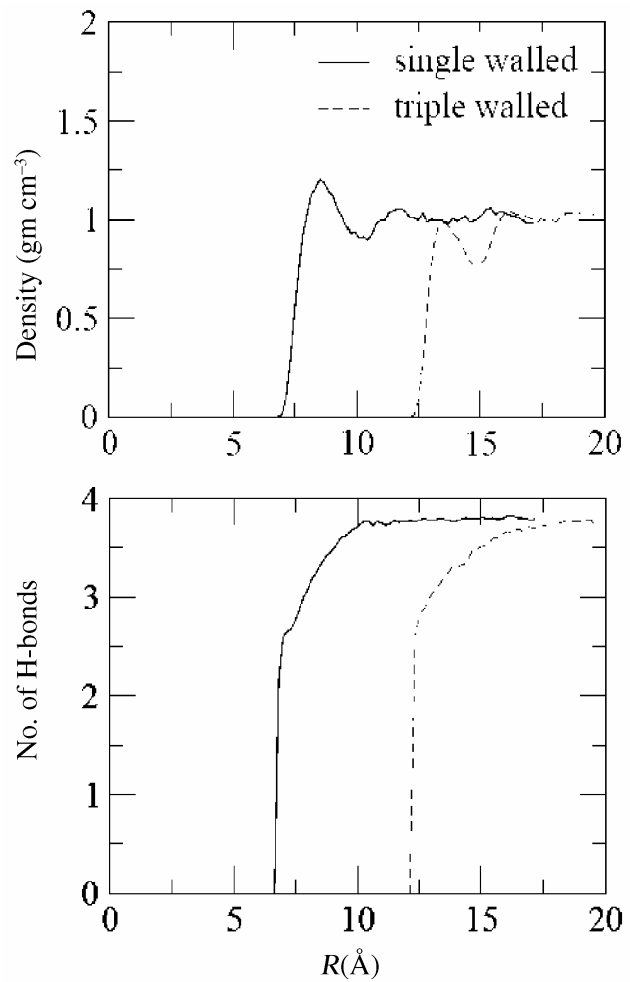

Figure 8. The density profiles and hydrogen bond distributions of water inside and in the vicinity of the outer surface of a single-walled and triple-walled $(6,6)$ nanotube for $\lambda=0$. and empty states, are more strongly influenced by the strength of the attractive dispersion interactions between the solute atoms and water molecules.

Since the solvation structure of narrow nanotubes are found to depend rather strongly on the nature of interaction potentials and the true interaction potential between a nanotube and water are not known, it is desirable to go beyond the use of empirical potentials and investigate these systems by means of $a b$ initio molecular dynamics simulations ${ }^{26}$ to understand the true nature of hydrophobic interactions between water and carbon nanotubes. Since such calculations are computationally much more expensive than the corresponding classical simulations, bigger systems of the type considered here are not suitable for $a b$ initio molecular dynamics calculations. Thus, it is important to know if the wetting/
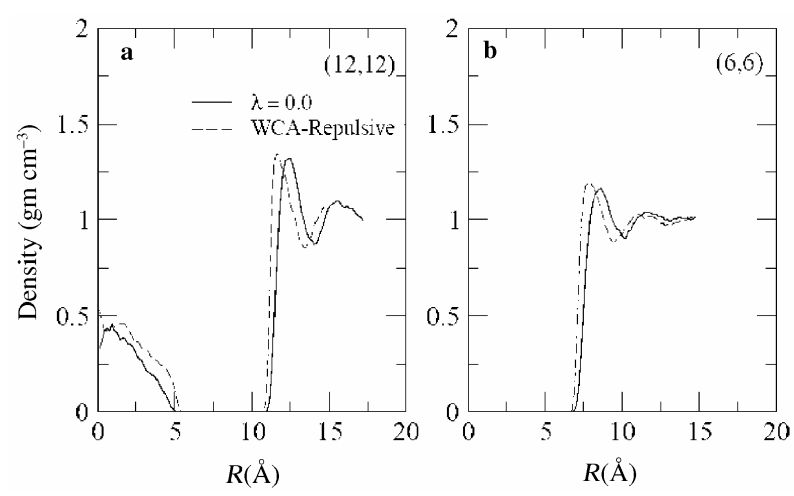

Figure 9. The density profiles of water inside and in the vicinity of the outer surfaces of single-walled $(6,6)$ and $(12,12)$ nanotubes for $\lambda=0$ and WCA-repulsive potentials.
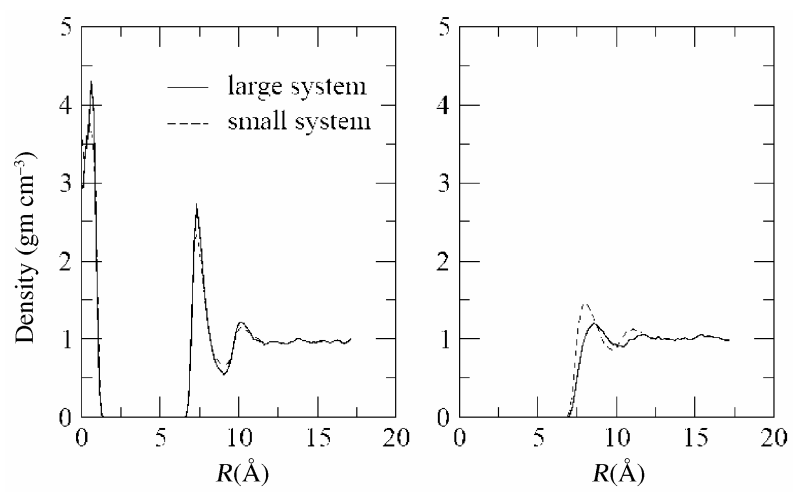

Figure 10. The density profiles of water inside and in the vicinity of the outer surface of a single-walled $(6,6)$ nanotube for two different system sizes and for $\lambda=1.0$ and $\lambda=0$. 
dewetting behaviour that is observed for the bigger systems will also hold for relatively smaller systems of, say, a few hundred molecules. In this regard, we have simulated a smaller system consisting of a $(6,6)$ nanotube immersed in 455 water molecules (after removing the overlapping molecules) and the results for the smaller system are shown in figure 10 for both $\lambda=1$ and $\lambda=0$. For comparison, we have also included the results of figure $3 \mathrm{~b}$ which were obtained for a larger system with the same nanotube. It is seen that the main characteristics of hydrophobic solvation, i.e. filling for $\lambda=1.0$ and emptying for $\lambda=0$, are also reproduced by the smaller system.

\section{Conclusions}

In this work, we have investigated the hydration characteristics of hydrophobic carbon nanotubes in water for different diameter, wall thickness and solutewater dispersive interactions. The focus was primarily on the filled versus empty states of the nanotubes as the diameter and interaction potential are changed. However, we have also looked at the density profiles near the outer surfaces and hydrogen bond distributions of water both inside and outside the nanotubes for all the systems considered here. Results are also presented for different size of the simulation systems. In particular, we have considered nanotubes of varying diameter immersed in liquid water with tunable strength of the attractive part of the carbon-water dispersion interaction. It is found that although the tuning of the attractive part of the interactions plays a minor role in the structure of water near a small carbon atomic solute, it plays a significant role in determining the filling of the nanotubes and also the structure of water near in the vicinity of nanotubes made of many such atoms. Depending on the radii of the nanotubes and strength of the attractive interactions, different behaviour from wetting to dewetting of the confined region inside the nanotubes is observed when the nanotubes are not too wide. Similar results are found for two different kinds of repulsive potentials employed in the present work. Our calculations with single-walled versus triple-walled nanotubes show that a variation in the thickness of the walls does not alter the filling behaviour, overall hydration characteristics and also hydrogen bond distributions in any significant manner. Although majority of our calculations are done with rather large systems consisting of about a thousand solvent molecules, some additional calculations with differ- ent sized systems reveal that smaller systems with a total of about four hundred solvent molecules can also exhibit the essentials of wetting and dewetting behaviour on changes of the attractive strength of the carbon-water interaction potential. We hope that, in near future, it would be possible to handle such smaller systems by means of ab initio molecular dynamics methods without involving any predefined empirical potentials so that a better understanding of the molecular nature of hydrophobic solvation in aqueous systems containing carbon nanotubes could be achieved from first principles.

\section{Acknowledgement}

We thank Department of Science and Technology (DST) and Council of Scientific and Industrial Research (CSIR), Government of India, for financial support and University Grants Commission (UGC) for a research fellowship to M R.

\section{References}

1. Gogotsi Y, Libera J A, Güvenc-Yazicioglu A and Megaridis C M 2001 Appl. Phys. Lett. 791021

2. Megaridis C M, Güvenc-Yazicioglu A, Libera J A and Gogotsi Y 2002 Phys. Fluids 14 L5; Megaridis C M, Güvenc-Yazicioglu A, Libera J A and Gogotsi Y 2001 Appl. Phys. Lett. 791021

3. Kolesnikov A I, Zanotti J, Loong C and Thiyagarajan P 2004 Phys. Rev. Lett. 93035503

4. Maniwa Y et al 2005 Chem. Phys. Lett. 401534

5. Lynden-Bell R M and Rasaiah J C 1996 J. Chem. Phys. 1059266

6. Lakatos G and Patey G N 2007 J. Chem. Phys. 126 024703

7. Brovchenko I, Geiger A and Oleinikova A $2004 \mathrm{~J}$. Phys.: Condens. Matter 16 S5353

8. Tang, Y W, Chan K-Y and Szalai I 2004 J. Phys. Chem. B108 18204

9. Gordillo M C and Marti J 2000 Chem. Phys. Lett. 329 341

10. Marti J and Gordillo M C 2001 J. Chem. Phys. 114 10486; Marti J and Gordillo M C 2003 J. Chem. Phys. 11912540

11. Mao Z and Sinnott S B 2000 J. Phys. Chem. B104 4618

12. Hanasaki I and Nakatani A 2006 J. Chem. Phys. 124 174714

13. Striolo A, Gubbins K E, Chialvo A A and Cummings P T 2004 Mol. Phys. 102243

14. Striolo A, Chialvo A A, Gubbins K E and Cummings P T 2005 J. Chem. Phys. 122234712

15. Hummer G, Rasaiah J C and Noworyta J P 2001 Nature (London) $\mathbf{4 1 4} 188$

16. Waghe A, Rasaiah J C and Hummer G 2002 J. Chem. Phys. 11710789 
17. Mukherjee B, Maiti P K, Dasgupta C and Sood A K 2007 J. Chem. Phys. 126124704

18. Zhu F and Schulten K 2003 Biophys. J. 85236

19. Berendsen H J C, Grigera J R and Straatsma T P 1987 J. Phys. Chem. 916269

20. Cornell D W et al 1995 J. Am. Chem. Soc. 117 5179

21. Chandler D, Weeks J D and Andersen H C 1983 Science 220787

22. Allen M P and Tildesley D J 1987 Computer simulation of liquids (New York: Oxford)
23. Choudhury N and Pettitt B M 2005 J. Am. Chem. Soc. 127, 3556; Choudhury N and Pettitt B M 2005 J. Am. Chem. Soc. 1294847

24. Luzar A and Chandler D 1996 Phys. Rev. Lett. 76 928; Luzar A and Chandler D 1996 Nature (London) 37955

25. Chowdhuri S and Chandra A 2006 J. Phys. Chem. B110 9674; Mallik B S and Chandra A 2006 J. Chem. Phys. 125234502

26. Dellago C and Naor M M 2005 Chem. Phys. Lett. 169 36 